\title{
MEDICAL TELEREHABILITATION: AN EFFECTIVE METHOD FOR THE LONG-DISTANCE FUNCTIONAL EVALUATION OF PATIENTS
}

\author{
Dogaru Gabriela ${ }^{12}$; Stanescu, Ioana ${ }^{12}$; Pasca Luminita ${ }^{1,3}$ \\ 1. "Iuliu Hatieganu" University of Medicine and Pharmacy Cluj-Napoca \\ 2. Clinical Rehabilitation Hospital Cluj-Napoca \\ 3. City Clinical Hospital Cluj-Napoca
}

Experts predicted as early as 10 years ago that starting with 2010, at least $15 \%$ of health care services worldwide would be provided from a distance, using telemedicine (1). The expansion of the fields of medicine at a distance has been possible due to the progress made in the area of communication and information technologies. These can increase the capacity of health care services, improve service provision and allow people to better take care of their health. In a society undergoing a significant transformation process at the beginning of the third millennium, the use of modern communication means for the improvement of the quality of life of disadvantaged social groups is a necessity (2).

The aim of medical rehabilitation is to improve individual functioning, to set the treatment objectives of lost function recovery, to plan and implement measures, as well as to identify the problems and needs of a person that lives in a certain environment, with the necessity of certain changes in that environment in order to solve the problems. Rehabilitation measures concern the functions and structures of the body, activities and social participation, environmental factors and personal factors. These contribute to providing and maintaining an optimal functioning of a person in the interaction with the environment, using the following general results: prevention of function loss; slowing of the function loss rate; improvement or recovery of function; compensation for the lost function; maintenance of the current function (5).

The development of new communication technologies has changed the way of thinking, action and communication in the health care system all over the world; thus, new areas as part of health care services have appeared: telehealth, telemedicine, and telerehabilitation.

- "Telehealth" ("e-health") refers to the use of electronic information and telecommunication technologies for facilitating long-distance health care services, medical education, and public health and sanitary administration problems (3). Most of the existing platforms are aimed at using technological instruments for the management of processes and communication between medical institutions and/or between various departments of these institutions or externalized departments.

- "Telemedicine" refers to the use of electronic information and telecommunication technologies only for providing health care services to the population. The applications of telemedicine are numerous and have found their utility in many medical and surgical specialties (cardiology, dermatology, neurosurgery, pathological anatomy, radiology or oncology).

- "Telerehabilitation" refers to the longdistance provision of medical rehabilitation services by means of new communication and information technologies (videotelephones, videoconferences, virtual reality, web and internet technology based applications, PDAs, even some types of robots) (4).

Some examples of telerehabilitation are:

-Services of telepsychiatry, cardiac rehabilitation, language and speech therapy, and cognitive therapy for persons with brain lesions.

-Long-distance evaluations in order to provide services of altering the living places of the elderly;

-Training and support of the staff in the medical area; 
-Computerized guiding principles to help doctors use adequate interventions;

-Consultation between tertiary care hospitals and community hospitals regarding prosthetic and orthotic problems and the recommendation of the wheelchair;

-Sharing professional experience between countries, as well as at critical times, such as in the wake of a disaster (5);

One of the premises that led to the appearance of long-distance rehabilitation was the change over the past decade in the perception of the health care service provision. The conception that high quality medical services can be provided only in hospitals is about to be replaced with the tendency to decentralize and externalize many health care services towards specialized ambulatory care units, family doctor offices, elderly care institutions or even patients' homes. This tendency is based on new information and communication technologies, without which telerehabilitation would not have been possible.

According to the World Report on Disability (5), blockages have been identified in the provision of medical rehabilitation services to the population, as there is an unfulfilled need for this type of services and an offer that does not satisfy the demand. The number of medical rehabilitation specialists, as well as the number of rehabilitation centers has proved to be insufficient compared to the number of demands. According to the statistics of the World Health Organization, by 2050 the number of persons aged over 65 years will increase by $73 \%$ in developed countries and by $207 \%$ globally; the demand for health care services is much higher in this category of patients, predisposed to a higher degree of disability due to age related diseases (cerebrovascular accidents, dementia, fractures).

More than one billion people worldwide live with a form of disability, which is about $15 \%$ of the world population, according to global population estimates of 2010. This figure exceeds the previous estimates of the
World Health Organization, which date back to the '70s and suggest a figure of about $10 \%$. More than 190 million people have severe disabilities such as tetraplegia, severe depression or blinding, according to the Global Burden of Disease Study ordered by the World Bank.

The estimated number of children (0-14 years) who live with disabilities varies between 93 millions and 150 millions (5).

This is why an increased pressure is exerted on health care and social service providers, which will have to keep up with the increasing numbers of elderly patients.

A very high pressure is also exerted on medical rehabilitation hospital units, which are required to provide increasingly better quality rehabilitation services to an increasing number of patients, under the conditions of decreasing financing. Confronted with these problems, rehabilitation centers have tried to reduce the mean hospitalization duration, to refer patients to nursing centers (with less complex services) or to simplify the rehabilitation techniques (for example, placing emphasis on the use of the healthy limbs, not on the recovery of the function of the paralyzed limb).

Another problem identified in relation to the provision of rehabilitation services to the population is the difficult access of some categories of patients to medical centers. Access is a problem for patients with motor disabilities, but also for those who live in difficult access or isolated areas, and transportation to a rehabilitation center situated at a distance from home can pose logistical as well as material problems.

A solution for overcoming barriers to access to rehabilitation services is provided by longdistance medical rehabilitation. This is a widely accessible service, developed for the community, which mainly benefits people with special needs who live at a distance from specialized medical services (for example, in isolated rural communities), people whose disabilities do not allow them to reach the hospital/specialized medical office, as well as patients who do not have in their residence area a rehabilitation center or a specialist in medical rehabilitation. These persons are provided with 
quality services in a relatively short time, which makes disappear the distance, time, cost and convenience barriers (6) between the doctor and a special category of patients: those with special needs and/or with disabilities. Through this method, the autonomy of patients at their homes is facilitated, and their institutionalization decreases.

Telerehabilitation provides the patients with the following long-distance services:

1.Functional evaluation: a). simple - by interview or use of questionnaires (e.g. for assessing the performance of activities of daily life - ADL) - which requires a low speed internet connection, and b). complex by the video assessment of motor or occupational performance - which requires a high speed internet connection and experienced clinicians (7).

2.Examination of the patient by the specialist in rehabilitation medicine or a multidisciplinary team, with the making of diagnosis and functional prognosis.

3.Elaboration of the intervention plan: setting the objectives to be reached in the rehabilitation process and the methods by which these objectives can be reached.

4.Provision at home of real-time or recorded kinesitherapy, speech therapy language reeducation and occupational therapy programs, using the patient's own computer, with the possibility of feedback from the kinesitherapist.

By means of telerehabilitation, the patient has the possibility to be examined and evaluated in real time both by the medical rehabilitation doctor and the physiotherapist or the speech therapist, with comparable results to those of a face-to-face consultation. Thus, the individual rehabilitation plan can be established by teams specialized in a certain field.

The quality of long-distance health care should be identical to that of a conventional consultation (8).

The system offers the patient the opportunity to participate in a real-time program of kinesitherapy and ergotherapy exercises performed by a physiotherapist, with the possibility of having a feedback of the correctness of the exercises performed. The kinesitherapist monitors on his own computer the way in which the program exercises are performed. The system also monitors the continuation of the kinesitherapy program at home by evaluating the patient at preestablished time intervals.

In addition to these methods, several types of complementary rehabilitation services have been developed, which can be provided to special categories of patients (persons with motor disabilities), using communication technologies:

- assistance in choosing assistive devices (crutches, walking frames, wheelchairs)

- training the patients to use them

- evaluation of the accessibility of the living place using virtual reality applications

- vocational rehabilitation - in which the abilities required for obtaining a certain job are assessed and exercised

A range of strategies is necessary to cover the lack of access of the disabled to the health care system, rehabilitation being one of the effective solutions (2).

The medical specialties that benefit from telerehabilitation are numerous: orthopedics (e.g. rehabilitation of patients after total hip or knee arthroplasty), rheumatology (e.g. rehabilitation of patients with chronic articular pain or persistent lumbar pain), neurology (speech rehabilitation, rehabilitation of the motor limb function lost following vascular accidents or trauma) - are just a few examples.

The technology used includes both classical vocal and visual communication methods using the computer, and new technologies, in which the therapist can guide the treatment from a distance using the internet (verbal communication connection) or even automated devices (robots), which allow the user to elaborate motor commands followed by the feedback of their execution.

By using the communication technology during medical consultations, rehabilitation at a distance or at the patient's home can be initiated for persons with physical disabilities whose access to such services is difficult for various reasons. 
However, accessibility to rehabilitation at a distance raises other barriers: the lack of standardized criteria for including patients in a telerehabilitation program - depending on their specific disorder, as well as the lack of clinical criteria for the long-distance evaluation of patients.

The results of a rehabilitation program are measured by the benefits gained in the functioning of an individual as a result of a set of rehabilitation measures. The results are also measured by evaluating the individual's social participation and performance in several areas: mobility, communication, self-care, education, work, quality of life. In the new field of telerehabilitation, the lack of criteria for assessing the efficiency of the methods used is a challenge that needs to be solved.

In conclusion, medical telerehabilitation consists of using information, communication and technologies in association with medical rehabilitation. This is a developing field that can increase the capacity and accessibility of rehabilitation measures by the long-distance provision of interventions.

\section{BIBLIOGRAPHY:}

1. Sinha, A. An overview of telemedicine: the virtual gaze of health care in the next century.Med Anthropol Q. 2000 Sep;14(3):291309. Review

2. Borde $A$ et al. Information and communication technologies for development: health. New York, Global Alliance for ICT and Development, 2010.

3. Schmeler MR, Schein M, Mc Cue M. Telerehab clinical and vocational Applications for Assistive Technology: Research, Opportunities, and Challenges, International Journal of Telerehabilitation, vol 1, nr 1, fall 2009.

4. Carignan CR, Krebs, HI, Telerehabilitation robotics: big lights, big future? Journal of Rehabilitation Research and Development, 2006; 43 (5): 695 - 710

5. Organizatia Mondiala a Sanatatii. Raport Mondial Privind Dizabilitatea, Ed Visual Promotion, 2012

6. McCue M, Fairman A, Pramuka M, Enhancing quality of life through telerehabilitation. Phys Med Rehabil Clin N Am. 2010 Feb;21(1):195-205

7. Wakeford, L et al, Telerehabilitation position paper. November/December 2005, Volume 59, Number 6, pag 656-660.

8. Agence d'évaluation des technologies et des modes d'intervention en santé (AETMIS). Telehealth: Clinical Guidelines and Technological Standards for Telerehabilitation. Report prepared by Gilles Pineau, Khalil Moqadem, Carole St-Hilaire, Robert Perreault, Éric Levac, and Bruno Hamel, with the collaboration of Hélène Bergeron, Alexandra Obadia and Lorraine Caron (AETMIS 06-03). Montréal: AETMIS, 2006, x p. 